\title{
Glioblastoma multiforme with oligodendroglial component (GBMO): favorable outcome after post-operative radiotherapy and chemotherapy with nimustine (ACNU) and teniposide (VM26)
}

\author{
Dirk Vordermark*1, Klemens Ruprecht ${ }^{2}$, Peter Rieckmann², \\ Wolfgang Roggendorf ${ }^{3}$, Giles H Vince ${ }^{4}$, Monika Warmuth-Metz ${ }^{5}$, \\ Oliver Kölbl ${ }^{1}$ and Michael Flentje ${ }^{1}$
}

\author{
Address: ${ }^{1}$ Dept. of Radiation Oncology, University of Würzburg, Würzburg, Germany, ${ }^{2}$ Dept. of Neurology, University of Würzburg, Würzburg, \\ Germany, ${ }^{3}$ Dept. of Neuropathology, University of Würzburg, Würzburg, Germany, ${ }^{4}$ Dept. of Radiosurgery, University of Würzburg, Würzburg, \\ Germany and ${ }^{5}$ Dept. of Neuroradiology, University of Würzburg, Würzburg, Germany \\ Email: Dirk Vordermark* - vordermark_d@klinik.uni-wuerzburg.de; Klemens Ruprecht - klemens.ruprecht@mail.uni-wuerzburg.de; \\ Peter Rieckmann - p.rieckmann@mail.uni-wuerzburg.de; Wolfgang Roggendorf - path060@mail.uni-wuerzburg.de; \\ Giles H Vince - gvince@gmx.de; Monika Warmuth-Metz - warmuth@neuroradiologie.uni-wuerzburg.de; \\ Oliver Kölbl - oliver.koelbl@klinik.uni-regensburg.de; Michael Flentje - flentje_m@klinik.uni-wuerzburg.de \\ * Corresponding author
}

Published: 18 October 2006

BMC Cancer 2006, 6:247 doi:10.1 |86/147|-2407-6-247
Received: 16 March 2006

Accepted: 18 October 2006

This article is available from: http://www.biomedcentral.com/I47I-2407/6/247

(C) 2006 Vordermark et al; licensee BioMed Central Ltd.

This is an Open Access article distributed under the terms of the Creative Commons Attribution License (http://creativecommons.org/licenses/by/2.0), which permits unrestricted use, distribution, and reproduction in any medium, provided the original work is properly cited.

\begin{abstract}
Background: The presence of an oligodendroglial component within a glioblastoma multiforme (GBM) is considered a prognostically favorable factor, but the clinical outcome of patients with glioblastoma multiforme with oligodendroglial component (GBMO) after combined post-operative radiotherapy and chemotherapy has rarely been reported.
\end{abstract}

Methods: We analyzed overall and progression-free survival in a group of ten consecutive patients initially diagnosed with GBMO between 1996 and 2004 (4.2\% of all GBM patients). Median (range) age was 54 (34-73) years, 90\% were resected and median radiotherapy dose was 54 (45-60.6) Gy. $80 \%$ of patients received post-operative chemotherapy with nimustine (ACNU) and VM26 (teniposide) for a median of $3.5(\mathrm{I}-6)$ cycles, the remainder were treated with post-operative radiotherapy alone. All specimens were reviewed by an experienced neuropathologist.

Results: Neuropathological re-evaluation revealed GBM with an oligodendroglial component of $30 \%$ or less in five cases, predominant oligoastrocytic tumors with focal areas of GBM in four patients and WHO grade III oligoastrocytoma with questionable transition to GBM in one patient. Four of ten patients were alive at at $40,4 \mathrm{I}, 4 \mathrm{I}$ and 82 months. The median overall survival (KaplanMeier) was 26 months, the 2-year survival rate was $60 \%$ (progression-free survival: 9.8 months and $40 \%$, respectively).

Conclusion: In conclusion, patients with GBMO treated with post-operative radiotherapy and chemotherapy with ACNU/VM26 had a better prognosis than reported for GBM in modern chemoradiation series. 


\section{Background}

Although glioblastoma multiforme with oligodendroglial component (GBMO) is not considered as a distinct disease entity in the most recent World Health Organization (WHO) classification of central nervous system tumors [1], the increased chemosensitivity and radiosensitivity of WHO grade III anaplastic oligodendrogliomas $[2,3]$ has led to the assumption that patients with GBMO might similarly have a better prognosis than patients with classic glioblastoma multiforme (GBM). To the authors' knowldedge, data from phase III clinical trials on the outcome of the subgroup of patients with GBMO have not been published. In trial 83-02 of the Radiation Therapy Oncology Group (RTOG), a four-arm randomized phase I/II trial assessing different radiotherapy fractionation schedules with carmustine (BCNU) chemotherapy in all arms, the median survival of 14.3 months in GBMO was significantly longer than the 10.4 months observed in classic GBM [4]. The 2-year survival rates were $35 \%$ and $4 \%$, respectively.

Until the recent establishment of combined chemoradiation with temozolomide as a new treatment standard [5], the use of chemotherapy in GBM patients was not generally recommended. A meta-analysis of randomized controlled trials of mostly nitrosurea-based chemotherapy in malignant glioma found an absolute increase in overall survival of $6 \%$ at one year and of $4 \%$ at two years in glioblastoma patients treated with chemotherapy [6]. In Germany, combination chemotherapy with nimustine (ACNU) and teniposide (VM26) was a preferred protocol in glioblastoma patients in whom chemotherapy was indicated, leading to a median overall survival of 17.3 months in glioblastoma in the NOA-01 trial [7]. It has been the policy in the authors' institution to recommend post-operative chemotherapy with ACNU and VM26, in addition to radiotherapy, if patients with GBMO were in sufficiently good general general condition (Karnofsky index of at least 70). We report here the clinical outcome of a series of ten consecutive patients with GBMO treated between 1996 and 2004.

\section{Methods}

\section{Patients and treatment}

The charts of all patients with a diagnosis of GBM referred to the Dept. of Radiation Oncology, University of Würzburg, were reviewed regarding a histopathological diagnosis of GBMO. For initial histopathological evaluation, immunohistochemistry for the markers glial fibirillary acidic protein (GFAP), Ki-67, p53 or CD68 was routinely performed by standard techniques. Although there is no uniform definition of GBMO, all patients in whom the initial histopathological report - on which treatment decisions were based - described a population of cells with oligodendroglial features next to a population of astrocytic and anaplastic glioma cells, with obvious features of highest malignancy typically found in GBM, were included in the study cohort, regardless of the extent of the oligodendroglial component [8]. Of 240 patients with GBM treated during the period studied, $\mathrm{n}=10(4.2 \%)$ were found to have an oligodendroglial component described in the initial pathology report. Histopathological slides of these ten patients were reviewed by an experienced neuropathologist (W. R.), who was blinded to the clinical outcome of the patients, on the basis of the WHO classification of 2000 [1]. The results of this re-evaluation did not affect inclusion of patients in the present analysis. The $1 \mathrm{p} / 19 \mathrm{q}$ status was not determined as part of the present study.

A summary of patient and treatment characteristics for this group of GBMO patients is given in Table 1. Characteristics of individual patients are shown in Table 2. Patients were assigned to defined prognostic groups based on the RTOG recursive-partioning analysis data for malignant glioma [9]. Treatment details are listed in Tables 1 and 2. Radiotherapy was administered as a 3-D conformal treatment, the target volume being based on contrastenhanced planning computed tomogrophy (CT) and preoperative imaging. Chemotherapy with ACNU $\left(90 \mathrm{mg} / \mathrm{m}^{2}\right.$ iv, day1) and VM26 (60 mg/m², days 1 to 3; both drugs given in six-week intervals) was usually initiated after completion of radiotherapy. In general, it was aimed to administer six cycles of chemotherapy. In three patients,

Table I: Overview of patient and treatment characteristics

\begin{tabular}{|c|c|c|}
\hline age at diagnosis (years) & median (range) & $54(34-73)$ \\
\hline surgical procedure & biopsy resection & $\begin{array}{l}1 / 10(10 \%) \\
9 / 10(90 \%)\end{array}$ \\
\hline total RT dose (Gy) & median (range) & $54(45-60.6)$ \\
\hline adjuvant chemotherapy with ACNU/VM26 & & $8 / 10(80 \%)$ \\
\hline no. of adjuvant cycles & median (range) & $3.5(1-6)$ \\
\hline$\geq 4$ adjuvant cycles & & $4 / 10(40 \%)$ \\
\hline
\end{tabular}

Overview of patient and treatment characteristics for $n=10$ consecutive patients with glioblastoma multiforme with oligodendroglial component (GBMO). 


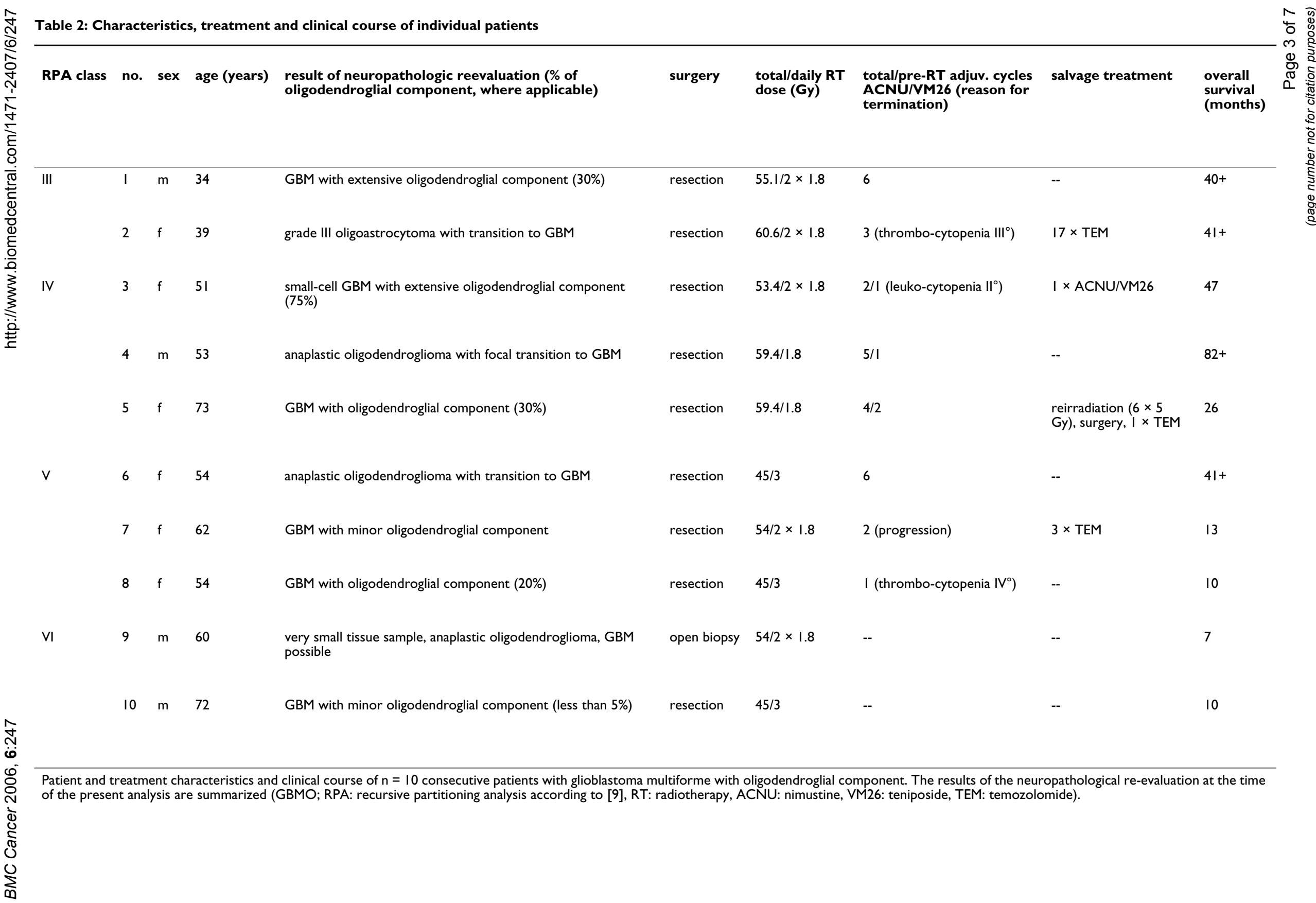


one or two cycles were given post-operatively before radiotherapy (Table 2).

\section{Statistics}

Overall survival was studied as the main end point. Overall survival status was obtained from local resident's registration offices. Progression-free survival was used as a secondary endpoint. The dates of diagnostic imaging studies were considered in the calculation of progression-free survival. Progression was defined as an unequivocal increase in the size of the contrast-enhancing lesion on CT or magnetic resonance imaging (MRI), typically performed in three-month intervals, leading to a consideration of salvage treatment options in the interdisciplinary tumor board. Actuarial survival rates were calculated from the time of histological diagnosis using the Kaplan-Meier method. Patients consented to anonymous data analysis as part of the treatment contract with the university hospital.

\section{Results}

The clinical course of individual patients is shown in Table 2. Eight of ten patients with GBMO received a median of 3.5 (range 1 to 6) cycles of chemotherapy with ACNU/VM26 as part of their adjuvant treatment. At the time of analysis, 4 of 10 patients were alive at 40, 41, 41 and 82 months. The Kaplan-Meier curves of overall survival and progression-free survival are shown in Fig. 1. The median overall survival of GBMO patients was 26 months. Overall survival at two years and at four years was $60 \%$ and $25 \%$, respectively. The median progression-free survival was 9.8 months. At two and four years, $40 \%$ and $26.7 \%$ of patients, respectively, were free of progression.

Histopathological re-evaluation of all specimens by an experienced neuropathologist revealed different degrees of oligodendroglial component within the tumor tissue. The grading of results of immunohistochemistry for GFAP, Ki-67, p53 and CD68 in individual patients is given in Table 3. Whereas five tumors were classified as GBM with an oligodendroglial component of $30 \%$ or less, the others were diagnosed as containing a predominant WHO grade III oligodendroglial or oligoastrocytic component. In four of the latter group, features of WHO grade IV GBM were seen. In one case, for which only very limited biopsy material was available, anaplastic oligodendroglioma was diagnosed upon review without confirmation of GBM. The overall survival times for patients with GBM and oligodendroglial component of $30 \%$ or less were $10,10,13$, 26 and $40+$ months as compared to $7,41+, 41+, 47$, and $82+$ months for patients with a predominant WHO grade III oligodendroglial or oligoastrocytic component.
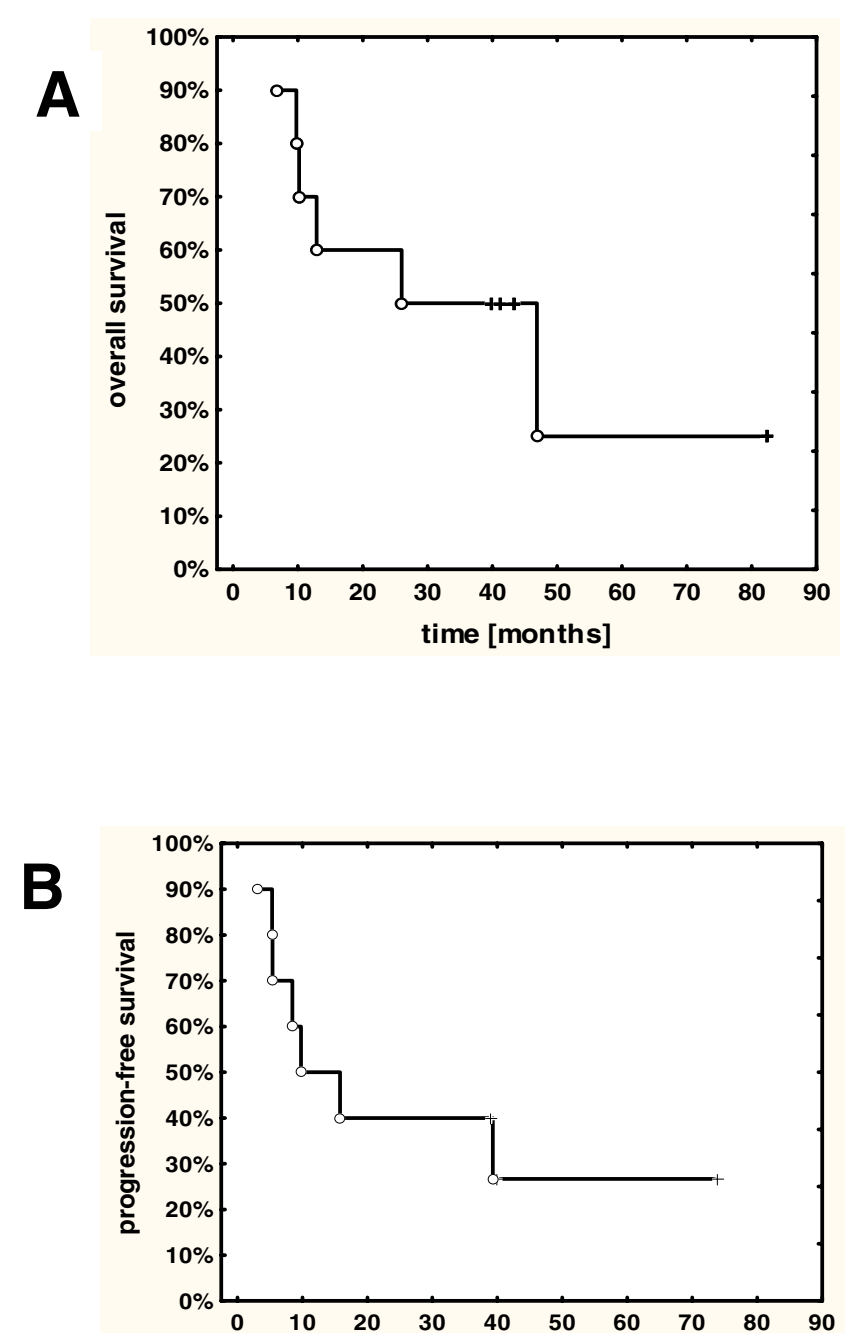

Figure I

Overall survival $(A)$ and progression-free survival $(B)$ of $n=$ I0 consecutive patients with glioblastoma multiforme with oligodendroglial component (GBMO).

\section{Discussion}

Before the recent introduction of combined radiochemotherapy with temozolomide [5], the use of chemotherapy in GBM was not universally recommended. The presence of an oligodendroglial component within an astrocytic tumor with features of a WHO grade IV glioblastoma, however, has been regarded by some authors as an indicator of increased chemosensitivity of such tumors. This assumption was based on the high response rates of WHO grade III anaplastic oligodendroglioma, as compared to grade III anaplastic astrocytoma, established in phase II trials in the 1990s [2]. Two recent randomized phase III trials of adjuvant PCV in anaplastic oligodendroglioma and anaplastic oligoastrocytoma, RTOG 94-02 and 
Table 3: Results of immunohistochemistry in individual patients with GBMO

\begin{tabular}{|c|c|c|c|c|}
\hline patient no. & GFAP & Ki-67 & p53 & CD68 \\
\hline I & ++ & + & ++ & n. a. \\
\hline 2 & + & +++ & + & ++ \\
\hline 3 & ++ & ++ & n. a. & + \\
\hline 4 & n. a. & n. a. & n. a. & n. a. \\
\hline 5 & ++ & + & n. a. & ++ \\
\hline 6 & + & +++ & n. a. & n. a. \\
\hline 7 & + & ++ & + & ++ \\
\hline 8 & + & ++ & + & ++ \\
\hline 9 & ++ & + & n. a. & ++ \\
\hline 10 & + & ++ & n. a. & n. a. \\
\hline
\end{tabular}

Results of immunohistochemistry for glial fibrillary acidic protein (GFAP), Ki-67, p53 and CD68 in individual patients with glioblastoma multiforme with oligodendroglial component (GBMO; patient numbers correspond to Table 2). Staining was scored as weak (+), intermediate $(++)$, strong $(+++)$ or not available (n.a.).

EORTC 26951, found a significant improvement in progression-free survival (2.6 vs. 1.9 years and 2.0 vs. 1.1 years, respectively) after addition of PCV chemotherapy, but so far no effect on overall survival $[10,11]$. The clinical outcome of patients with WHO grade IV GBMO has rarely been reported. Median survival rates in the small series available in the literature (Table 4) ranged between 14 and 26 months, 2-year survival rates between $20 \%$ and $58 \%$. The overall survival of 10 consecutive patients with GBMO in the present series (median 26 months, 60\% at two years) compares favorably to these GBMO series and to the outcome of all GBM patients in large clinical trials, including the temozolomide radiochemotherapy arm in EORTC 26981 and the ACNU/VM26 arm in the NOA-01 trial $[5,7]$.

The favorable survival rates in the present series of patients with GBMO may be a result of a good response of these tumors to initial multi-modality management with surgery, post-operative irradiation and chemotherapy with ACNU/VM26 (where feasible), but may also be influenced by the criteria for patient selection and the response to salvage therapy. Although the patients now analyzed were retrieved from the database of the radiation oncology department it appears unlikely that this introduced a relevant selection bias. Post-operative radiotherapy is a standard treatment in GBM and the reported median survival rate of all patients with GBM treated with radiotherapy in this department between 1990 and 1999 was 11.5 months [12]. The term GBMO has not been uniformly defined and especially the distinction of GBMO from anaplastic oligoastrocytoma is generally considered difficult $[1,8,13]$. However, nine of ten patients included clearly had tumors with components of highest malignancy, as found in grade IV GBM. In one patient with very limited biopsy material, the GBM component within a grade III oligoastrocytoma was judged as uncertain upon review. The fact that the patients now analyzed were actually considered to have GBM at the time of adjuvant treatment planning is underscored by the use of accelerated radiotherapy schedules, limiting the treatment duration to approximately three weeks, in 8 of 10 patients - an approach reserved for grade IV tumors at the authors' institution.

Within the limited patient cohort studied, the extent of the oligodendroglial component appeared to be linked to prognosis. Whereas only one of five patients with $30 \%$ or

Table 4: Treatment results of selected series of GBM and GBMO

\begin{tabular}{|c|c|c|c|c|c|c|}
\hline series & patient group & $\mathbf{n}$ & treatment & $\begin{array}{c}\text { median overall } \\
\text { survival (months) }\end{array}$ & $\begin{array}{c}\text { 2-year overall } \\
\text { survival }\end{array}$ & reference \\
\hline NOA-OI & all GBM & 154 & post-OP RT + ACNU/VM26 & 17.3 & $25 \%$ & [7] \\
\hline EORTC 2698I & all GBM & 287 & post-OP RT + TEM & 14.6 & $26.5 \%$ & {$[5]$} \\
\hline RTOG 83-02 & GBMO & 23 & post-OP RT + BCNU & 14.3 & $\begin{array}{c}\text { (3-year survival: } \\
35 \%)\end{array}$ & {$[4]$} \\
\hline retrospective & GBMO & 12 & resection $+\mathrm{RT}$, chemo in $2 / 12$ & 26 & $\begin{array}{l}58 \% \text { (data taken } \\
\text { from graph) }\end{array}$ & [8] \\
\hline retrospective & GBMO & II & post-OP RT in $65 \%$ (total group) & 17.7 & $\begin{array}{l}20 \% \text { (data taken } \\
\text { from graph) }\end{array}$ & {$[18]$} \\
\hline retrospective & GBMO & 5 & post-OP RT in $80 \%$ & 16.2 & n. a. & [19] \\
\hline present series & GBMO & 10 & post-OP RT + ACNU/VM26 (8/I0) & 26 & $60 \%$ & \\
\hline
\end{tabular}

Overview of treatment results in glioblastoma multiforme (GBM) and GBM with oligodendroglial component (GBMO) from selected series (RT: radiotherapy, ACNU: nimustine, VM26: teniposide, TEM: temozolomide, n. a.: not available). 
less oligodendroglial cells survived for more than two years, this was true for four of five patients with a predominant oligodendroglial component. Obviously, such quantifications must be regarded with caution as percentages for particular cell types on sectioned material need not be representative of the overall tumors.

GBMO tumors have been reported to frequently contain molecular genetic alterations associated with increased chemosensitivity in oligodendroglial tumors such as a loss of heterozygosity (LOH) on chromosome arms $1 \mathrm{p}$ and $19 \mathrm{q}[8,13]$. Microarray analysis of gene expression has been suggested as a method to distinguish "classic GBM" tumors from a group termed "non-classic" GBM with a better prognosis [14]. In the present series, identification of an oligodendroglial component on routine histopathological evaluation of tumor sections was apparently able to select a patient group with a better prognosis than in classic GBM.

In some of the previous series of patients with GBMO, patients were mostly treated with surgery and post-operative radiotherapy, but without chemotherapy. This may in part explain the favorable results of the present analysis. The low rate of patients surgically treated with biopsy only $(1 / 10$ in the present series) is comparable to the series of Kraus et al. [8] (Table 3) where all patients were resected such that a particularly aggressive surgical approach in the present patient group appears to be an unlikely explanation of the high survival rates.

The progression-free survival observed may be regarded as a better indicator of the efficacy of the initial treatment regimen. The median progression-free survival of 9.8 months is also longer than that seen in large trials of GBM, e. g. 6.9 months in the temozolomide arm of EORTC 26981 [5]. This suggests that the response of GBMO to ACNU/VM26 might be better than that of GBM in general to temozolomide. Clearly, the use of salvage treatments including surgery, stereotactic hypofractionated reirradiation [15] and temozolomide chemotherapy has led to prolonged survival in individual patients failing early after initial post-operative chemotherapy with ACNU/VM26 (Table 2). The efficacy of temozolomide as an initial treatment or as salvage therapy for GBMO has not been published to our knowledge.

The information gained by the present study is limited by the small number of patients who, nevertheless, represent the total number of this entitity from an eight-year period at a university center. A similar analysis focussing on specific effects of the addition of chemotherapy in patients with GBMO would be possible in subsets of patients from recently completed randomized trials in glioblastoma. It would, therefore, be of interest to analyze specifically the clinical course of GBM patients with an oligodendroglial component treated with temozolomide in EORTC 26981. It is also unknown whether patients with GBMO might benefit from an escalation of radiation dose, an approach currently considered not benefitial in GBM [16], or from high-dose chemotherapy protocols as investigated in anaplastic oligodendroglioma [17].

\section{Conclusion}

In summary, the prognosis of patients with GBMO treated with post-operative radiotherapy and chemotherapy with ACNU/VM26 was favorable compared to published data for GBMO treated mostly with post-operative radiotherapy alone and compared to GBM treated with post-operative radiotherapy and different regimens of chemotherapy. The outcome of GBMO after radiotherapy with concomitant and adjuvant temozolomide, the current standard for GBM, is not known. A superiority of ACNU/VM26 over temozolomide in GBMO can not be advocated. From our experience, post-operative chemotherapy should be offered to patients with GBM whenever possible in the presence of an oligodendroglial component.

\section{Competing interests}

The author(s) declare that they have no competing interests.

\section{Authors' contributions}

DV designed the analysis, reviewed patient data, performed statistical analysis and drafted the manuscript.

KR treated the patients, reviewed patient data and revised the manuscript.

PR treated the patients, reviewed patient data and revised the manuscript.

WR performed neuropathological evaluation and revised the manuscript.

GHV treated the patients, reviewed patient data and revised the manuscript.

MWM performed imaging studies on the patients, reviewed patient data and revised the manuscript.

OK treated the patients, reviewed patient data and revised the manuscript.

MF treated the patients, reviewed patient data and revised the manuscript.

All authors read and approved the final manuscript. 


\section{References}

I. Kleihues P, Louis DN, Scheithauer BW, Rorke LB, Reifenberger G, Burger PC, Cavenee WK: The WHO classification of tumor of the nervous system. J Neuropathol Exp Neurol 2002, 6 I:21 5-225.

2. Cairncross G, Macdonald D, Ludwin S, Lee D, Cascino T, Buckner J, Fulton D, Dropcho E, Stewart D, Schold C Jr, et al.: Chemotherapy for anaplastic oligodendroglioma. National Cancer Institute of Canada Clinical Trials Group. I Clin Oncol I 994, I 2:2013-202 I.

3. Heesters M, Molenaar W, Go GK: Radiotherapy in supratentorial gliomas. Strahlenther Onkol 2003, I 79:606-I4.

4. Nelson JS, Petito CK, Scott CB, Rotman M, Asbell S, Murray K Glioblastoma with oligodendroglial features (GBM-OL): report from Radiation Therapy Oncology Group (RTOG) trial 8302 [abstract]. Lab Invest 1996, 75:|4|A

5. Stupp R, Mason WP, van den Bent MJ, Weller M, Fisher B, Taphoorn MJ, Belanger K, Brandes AA, Marosi C, Bogdahn U, Curschmann J, Janzer RC, Ludwin SK, Gorlia T, Allgeier A, Lacombe D, Cairncross JG, Eisenhauer E, Mirimanoff RO, European Organisation for Research and Treatment of Cancer Brain Tumor and Radiotherapy Groups; National Cancer Institute of Canada Clinical Trials Group: Radiotherapy plus concomitant and adjuvant temozolomide for glioblastoma. N Engl J Med 2005, 352:987-996.

6. Stewart LA: Chemotherapy in adult high-grade glioma: a systematic review and meta-analysis of individual patient data from 12 randomised trials. Lancet 2002, 359: $1011-1018$.

7. Weller M, Muller B, Koch R, Bamberg M, Krauseneck P, NeuroOncology Working Group of the German Cancer Society: NeuroOncology Working Group 0 I trial of nimustine plus teniposide versus nimustine plus cytarabine chemotherapy in addition to involved-field radiotherapy in the first-line treatment of malignant glioma. J Clin Oncol 2003, 2 I:3276-3284.

8. Kraus JA, Lamszus K, Glesmann N, Beck M, Wolter M, Sabel M, Krex D, Klockgether T, Reifenberger G, Schlegel U: Molecular genetic alterations in glioblastomas with oligodendroglial component. Acta Neuropathol 200 I, I 0 I:3 I I-320.

9. Scott CB, Scarantino C, Urtasun R, Movsas B, Jones CU, Simpson JR, Fischbach AJ, Curran WJ Jr: Validation and predictive power of Radiation Therapy Oncology Group (RTOG) recursive partitioning analysis classes for malignant glioma patients: a report using RTOG 90-06. Int J Radiat Oncol Biol Phys 1998, 40:5I-55.

10. van den Bent MJ, Delattre J, Brandes AA, Taphoorn MJ, Hoang Xuan K, Bernsen H, Frenay M, Grisold W, Gorlia T, Lacombe D: First analysis of EORTC trial $2695 \mathrm{I}$, a randomized phase III study of adjuvant PCV chemotherapy in patients with highly anaplastic oligodendroglioma [abstract]. I Clin Oncol 2005, 23(suppl):A-I503.

II. Cairncross G, Seiferheld W, Shaw E, Jenkins R, Scheithauer B, Brachman D, Buckner J, Fink K, Souhami L, Curran W Jr: An intergroup randomized controlled clinical trial (RCT) of chemotherapy plus radiation (RT) versus $R T$ alone for pure and mixed anaplastic oligodendrogliomas: initial report of RTOG 94-02 [abstract]. J Clin Oncol 2004, 23(suppl):A-I 500.

12. Thiele W, Zunterer H, Maessen D, Flentje M, Oppitz U: Conventional vs. accelerated CT-planned irradiation of glioblastoma multiforme [abstract]. Radiother Oncol 2000, 56(suppl I):27.

13. He J, Mokhtari K, Sanson M, Marie Y, Kujas M, Huguet S, Leuraud P, Capelle L, Delattre JY, Poirier J, Hoang-Xuan K: Glioblastomas with an oligodendroglial component: a pathological and molecular study. I Neuropathol Exp Neurol 2001, 60:863-87I.

14. Nutt CL, Mani DR, Betensky RA, Tamayo P, Cairncross JG, Ladd C, Pohl U, Hartmann C, McLaughlin ME, Batchelor TT, Black PM, von Deimling A, Pomeroy SL, Golub TR, Louis DN: Gene expressionbased classification of malignant gliomas correlates better with survival than histological classification. Cancer Res 2003, 63:1602-1607

I5. Vordermark D, Kölbl O, Ruprecht K, Vince GH, Bratengeier K, Flentje M: Hypofractionated stereotactic re-irradiation: treatment option in recurrent malignant glioma. BMC Cancer 2005, 5:55.

16. Vordermark D, Kölbl O: Lack of survival benefit after stereotactic radiosurgery boost for glioblastoma multiforme: randomized comparison of stereotactic radiosurgery followed by conventional radiotherapy with carmustine to conventional radiotherapy with carmustine for patients with glioblastoma multiforme: report of radiation therapy oncology group 93-05 protocol: in regard to Souhami et al. (Int J Radiat Oncol Biol Phys 2004;60:853-860). Int J Radiat Oncol Biol Phys 2005, 62:296-297.

17. Abrey LE, Childs BH, Paleologos N, Kaminer L, Rosenfeld S, Salzmann D, Finlay JL, Gardner S, Peterson K, Hu W, Swinnen L, Bayer R, Forsyth P, Stewart D, Smith AM, Macdonald DR, Weaver S, Ramsey DA, Nimer SD, DeAngelis LM, Cairncross JG: High-dose chemotherapy with stem cell rescue as initial therapy for anaplastic oligodendroglioma. J Neurooncol 2003, 65:127-I34.

18. Pinto LW, Chimelli L: Oligodendroglial and neuronal component in glioblastomas: possible relation with prognosis [Portuguese]. Arq Neuropsiquiatr 2004, 62:1074-1078.

19. Hilton DA, Penney M, Pobereskin L, Sanders H, Love S: Histological indicators of prognosis in glioblastomas: retinoblastoma protein expression and oligodendroglial differentiation indicate improved survival. Histopathology 2004, 44:555-560.

\section{Pre-publication history}

The pre-publication history for this paper can be accessed here:

http://www.biomedcentral.com/1471-2407/6/247/pre pub
Publish with Biomed Central and every scientist can read your work free of charge

"BioMed Central will be the most significant development for disseminating the results of biomedical research in our lifetime. "

Sir Paul Nurse, Cancer Research UK

Your research papers will be:

- available free of charge to the entire biomedical community

- peer reviewed and published immediately upon acceptance

- cited in PubMed and archived on PubMed Central

- yours - you keep the copyright

Submit your manuscript here:

http://www.biomedcentral.com/info/publishing_adv.asp
BioMedcentral 